\title{
OLFACTORY BULB RESPONSES IN THE TURTLE, WITH SPECIAL REFERENCE TO THE DEEP NEGATIVE SPIKE
}

\author{
Y. IWASE* AND D. LISENBY \\ Department of Zoology, Washington University, St. Louis, Missouri, U.S. A.
}

The olfactory bulb response following mucosal, nerve, or direct stimulation has been extensively examined in the frog $^{12}$ and in the rabbit ${ }^{5,6,15)}$ but no detailed description of a response ascribable to the apical dendrites of the mitral cells has been reported. The peripheral dendrites of these cells differ morphologically from the apical dendritic processes of the neocortex. The synaptic regions are restricted to the terminal branches of the dendrites, the region of the glomerulus. This circumstance makes them favorable structures for the investigation of physiological properties of the dendritic response. The mitral cell response in the turtle has been examined recently ${ }^{10,11}$ and was chosen here for further analysis for several reasons. The olfactory nerves run as a compact bundle and can be easily stimulated electrically, allowing for a well controlled orthodromic activation of the olfactory bulb neurones. Also, this species has only one large cell type, the mitral cell, as a secondary olfactory neurone, eliminating a confusion with responses from different cell types. which are located in the same area in the mammalian olfactory bulb. The reptilian bulb shows other distinctive features. The apical dendritic shaft of the mitral cell sends dendritic processes to more than one glomerulus. The mitral cells are not distributed in a compact layer but exhibit some degree of vertical scatter. A cell type called the goblet cell, possibly equivalent to the mammalian tufted cell, is located in the internal granular layer, and like the tufted cell found in mammals, its axons enter the anterior commissure. ${ }^{4,9)}$

\section{METHODS}

The species of turtle used in this investigation was Pseudemys elegans. The olfactory nerves and bulbs were exposed bilaterally by opening the skull with a dental drill. The dura was cut away and the arachnoid was carefully torn apart with fine forceps. This last procedure was carried out under a microscope to prevent excessive

Received for publication February 16, 1965

* 岩瀬善彦, Present address: Department of Physiology, Kyoto Prefectural University of Medicine, Kyoto, Japan 
damage to the blood vessels lying immediately under the arachnoid membrane. The jugular vein was cannulated, and Flaxedil diluted 1:4 with Ringer's solution was administered to achieve muscular paralysis. A total dose of $2-3 \mathrm{mg} / \mathrm{kg}$ was required to prevent movement. The trachea was sectioned and two polyethylene tubes were inserted into the lungs. Oxygen and $\mathrm{CO}_{2}(95 \%-5 \%)$ were then insufflated continuously. The surface of the exposed bulb was covered with Ringer's solution and a spatula was lightly pressed against the cortex to prevent pulsation.

All potential recording was done with glass micropipettes filled with $3 \mathrm{M} \mathrm{KCl}$ or $\mathrm{NaCl}$ having an electrical resistance of $10-20$ megohms. The recording microelectrode was connected to a high impedance cathode follower-type probe. Electrical activities were then amplified through a Grass condenser coupled preamplifier (single side to ground, input half amplitude frequencies 0.3 cycles and 30 kilocycles) and displayed on a Tektronix 502 oscilloscope. The decay time constant for the total amplifying system was $230 \mathrm{msec}$.

Orthodromic responses were evoked by stimulating the olfactory nerve through bipolar silver electrodes constructed of wire $0.15 \mathrm{~mm}$ in diameter. Two parallel steel wires $0.1 \mathrm{~mm}$ in diameter insulated except the tips were inserted into the contralateral bulb to stimulate the tested bulb via the interbulbar system, the anterior commissure. Due to difficulty in selective stimulation of the anterior commissure, the stimulating site was chosen at the middle-posterior region of the bulb, which also often resulted in activation of olfactory tract fibers. Bipolar silver ball-type electrodes, $1 \mathrm{~mm}$ in diameter, were used to stimulate the olfactory tract which is difficult to locate and for this reason the electrodes were placed in the pyriform cortex, where part of the tract terminates.

In depth recordings the depth reported in each case was the distance traveled by the micropipette after surface contact. From this figure the electrode tip cannot be precisely localized with respect to neural layers in the bulb due to a slight, but uncontrollable, surface dimpling and to regional differences in the actual thickness of the layers, particularly of the olfactory nerve layer which is relatively thick in the anterior region and decreases in thickness posteriorly. To avoid the latter uncertainty, records were usually led from the middle of the bulb where the depth of each layer had been determined histologically. However, even with this precaution, it was impossible to directly know the precise location of the electrode tip in the mitral cell layer, due to the previously mentioned vertical scatter of the mitral cells within that layer. ORREGO ${ }^{10)}$, using gross electrodes, had located in the turtle the sources of certain prominent features of the response by histological examination of electrode tip loci stained by the ferrocyanide technique. Similar response features serve here to identify physiologically the corresponding depths as reference levels.

\section{RESULTS}

In FIG. 1 a schematic representation of the neural elements of the olfactory bulb is presented along with records of the orthodromic cortical response led from the cortical surface and various layers. The orthodromic cortical response (OCR) recorded from the dorsal surface of the olfactory bulb consists of two surface-negative potentials. We shall call the first the initial potential and the second the slow potential. In the depth records a third potential appears, to be referred to as the deep spike. The initial potential disappears as the electrode is lowered deep into the bulb and the deep spike is recorded as a 
fast negative potential of 3 to $5 \mathrm{msec}$ duration, having a latency of $15 \mathrm{msec}$ as measured from the onset of the initial potential. At the upper level where the deep spike is recorded in isolation a positive deflection occurs, interpretable as the deep reflection of the slow negative wave in the surface record. Antecedent to this slow positive deflection the deep spike is depicted as a sharp negativity, sometimes accompanied by a preceding transient positive deflection. At the depth of $0.5 \mathrm{~mm}$, which would locate the electrode in the mitral cell layer, a single unit discharge, positive in sign, was seen to occur at approximately the same point in time where the deep spike started. Following this the negative deflection occurred, which had a steep onset and a slower return. It seems that this deflection is a compound potential of the deep spike and slow negativity, since both potentials are often recorded in an isolated form (cf. FIG. 2A).

FIG. 2A shows the deep spike recorded at the level of upper layer (external plexiform layer). The deep spike appeared in this experiment between the depths of 0.3 and $0.5 \mathrm{~mm}$, reaching a maximal amplitude at $0.4 \mathrm{~mm}$. The deep spike showed no increase in latency $(20 \mathrm{msec})$ or peak time until a depth of $0.5 \mathrm{~mm}$ was reached, at which point both values suddenly increased.
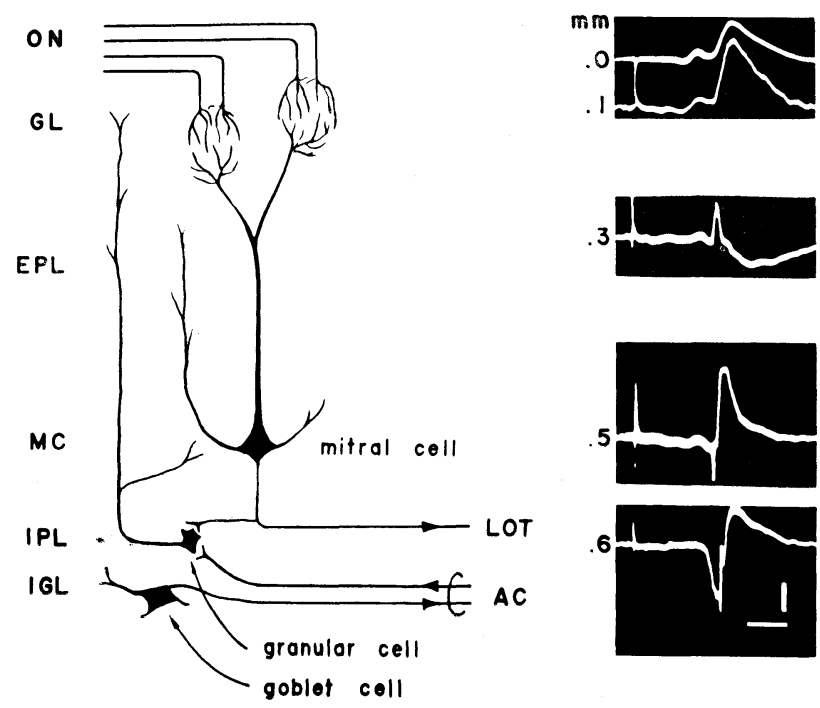

FIG. 1. A schematic drawing of the neural elements of the olfactory bulb and the orthodromic cortical response (OCR) recorded at the depths ranging from the surface to the internal granular layer. ON: olfactory nerve layer, GL: glomerular layer, EPL: external plexiform layer, MC: mitral cell layer, IPL: internal plexiform layer, IGL; internal granular layer. Recording site: middle region of the bulb; Left margin: cortical depths indicated in $\mathrm{mm}$; calibrations: $3 \mathrm{mV}$ and $20 \mathrm{msec}$. In this and subsequent figures upward deflection denotes negativity. 
FIG. 2B illustrates the recovery process of the deep spike, recorded at the level of maximum amplitude. This depth was $0.6 \mathrm{~mm}$, locating the electrode in the middle portion of the external plexiform layer in the anterior region of the bulb. The initial potential is recorded in low amplitude at this depth, because of the thickness of the olfactory nerve layer. This probably indicates that in control the initial potential has been lowered in size by the sharp positive deflection preceding the de€p negative spike. Then initial potentials in test responses are seen as being above control amplitude when the deep spike does not appear in the test response. The response to the test shock at intervals of $34 \mathrm{msec}$ or less consists mainly of the activity in nerve axons, as judged by the latency of the deflection and the rapid recovery as compared with that of the deep spike. Following this the deep spike increased gradually beyond a $0.13 \mathrm{sec}$ interval. Full recovery of the amplitude of the deep spike following olfactory nerve stimulation requires more than $2.0 \mathrm{sec}$.

An interaction experiment between the OCR and the commissural cortical

A

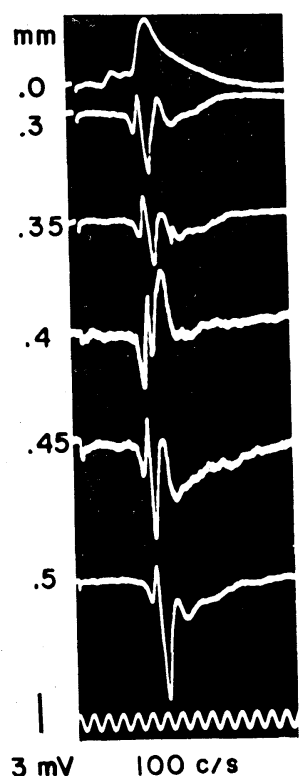

B

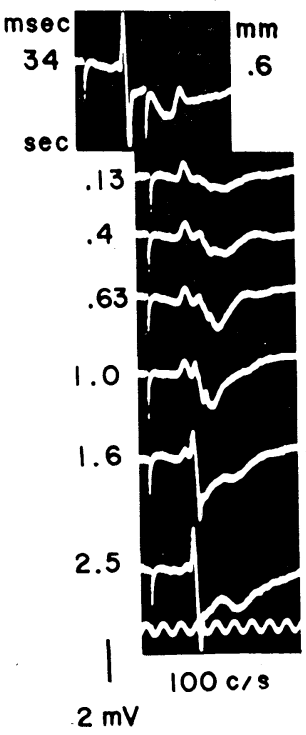

FIG. 2. A: The deep spike recorded at the level of external plexiform layer. Left margin; cortical depth in $\mathrm{mm}$, voltage calibration; $3 \mathrm{mV}$. B: Recovery of the deep spike. Recording site; anterior region of the bulb. Top record shows the conditioning and test responses at shock intervals of $34 \mathrm{msec}$. The following records show only the test response at shock intervals indicated beside each figure in sec. 
response $(\mathrm{CCR})$ is shown in FIG. 3. Both responses were recorded with the same electrode situated at a depth, probably the external plexiform layer, which yielded the highest amplitude of the deep spike to orthodromic stimulation. Although the CCR can be more clearly recorded at deeper levels, the external plexiform layer was chosen because the most important potential in this experiment was the deep spike. The control OCR consisted of an isolated deep spike followed by a slower negativity which was superimposed on a positive deflection mirroring the surface negative slow potential. The control CCR was composed of two deflections. The first, immediately following the stimulus artifact, was the response in the bulb on the stimulated side which spread electrotonically into the recording site (FIG. 4B). This deflection did not vary in latency or pattern with changes in the recording position. The second deflection had a latency of about $60 \mathrm{msec}$ and was partially summatable with double shocks. It was the true response in the tested bulb to contralateral stimulation and consisted of a slightly positive deflection followed by a larger and prolonged negative one (FIG. 3). During the slow negative excursion of the CCR the deep spike was slightly depressed. This attenuation was so slight as to be almost imperceptible. However, the slow negativity of the OCR was markedly attenuated. Another experiment of this interaction shows that the size of the slow negativity in the OCR decreased greatly during the excursion of the CCR and increased markedly for about $200 \mathrm{msec}$ after the CCR.

Stimulation of the homolateral pyriform cortex induced the antidromic

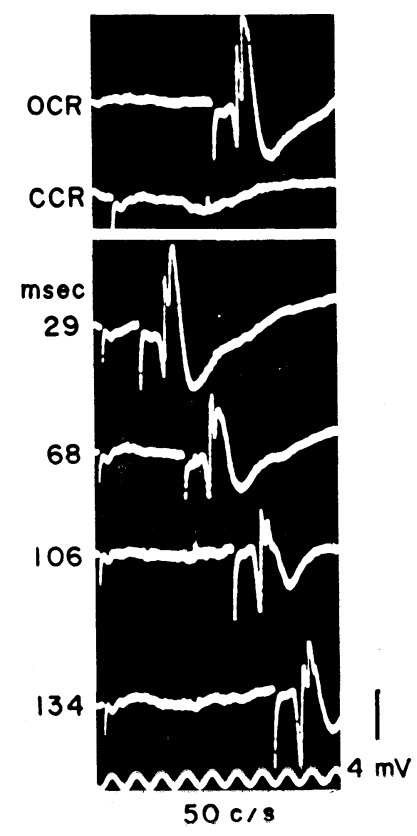

FIG. 3. Interaction between the orthodromic cortical response (OCR) and the commissural cortical response (CCR). Recording depth : $0.4 \mathrm{~mm}$. The top record is the control for both responses. Contralateral stimulation precedes the orthodromic stimulation at shock intervals indicated in left margin in msec. 
response (ACR) illustrated in FIG. 4A. The recording depth in each case was determined by the known characteristics of the orthodromic response. When the deep spike induced by an orthodromic stimulus reached its maximal amplitude, earlier determinations indicated that the electrode was situated in the external plexiform layer. The appearance of orthodromic unit discharges indicated the depth of the mitral cell layer, and phase reversal of the deep spike showed that the electrode was in the internal plexiform of internal plexiform or internal granular layer. At each level an antidromic response was evoked and recorded before advancing the electrode deeper. In the external plexiform layer an antidromic stimulus produced a slow long-lasting negativity preceded by an initial positivity. In the mitral cell layer the antidromic cortical response showed a decrease in amplitude and peak time, and a single unit discharge with a latency of about $20 \mathrm{msec}$ is seen at the initiation point of the slow negativity. This negativity became even smaller in the internal plexiform layer and a group of small spike potentials was recorded preceding it. The latency of the first spike in this envelope was $13 \mathrm{msec}$. Antidromic stimulation, therefore, first induced the grouped spikes recorded at the deepest level, the internal plexiform layer, then a unit discharge in the mitral cell layer and finally a slow negativity like that seen most clearly following orthodromic
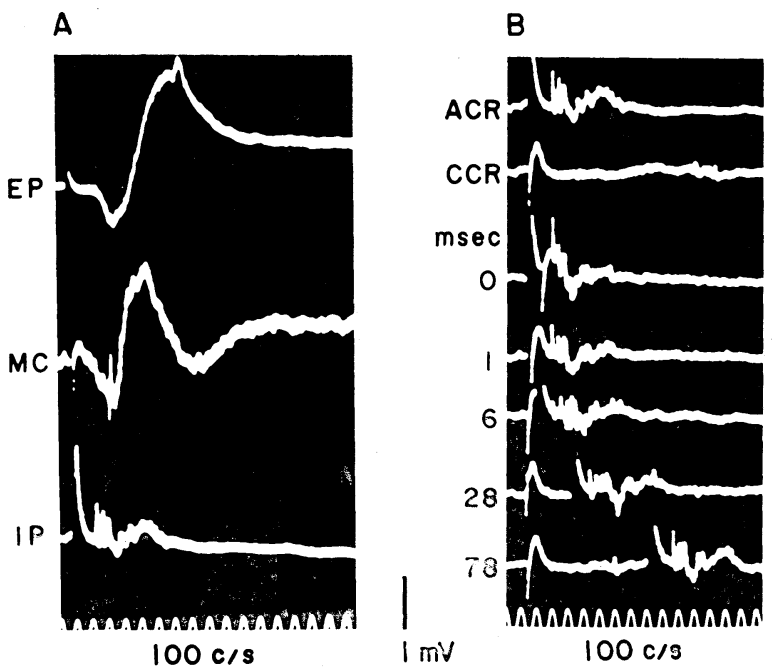

FIG. 4. Antidromic cortical response (ACR) and interaction between the ACR and the commissural cortical response (CCR). A : Recording of the ACR at different depths in the olfactory bulb. EP; external plexiform layer, MC; mitral cell layer, IP; internal plexiform layer. B: Control ACR and CCR at top with an interaction experiment below. Control stimulus precedes antidromic stimulus by interval indicated in msec. Recorded in internal layer. 
stimulation in the external plexiform layer, but here assignable to the level of the mitral cell bodies.

An example of interaction between the ACR and CCR is illustrated in FIG. 4B. In this case, contralateral stimulation preceded antidromic stimulation. The spike group of the ACR was superimposed on the CCR with no change in amplitude, indicating no interaction. The negativity which followed the $\mathrm{ACR}$ spike group was depressed during the excursion of the CCR but only slightly.

Two examples of interaction between the ACR and $\mathrm{OCR}$ are illustrated

$A$

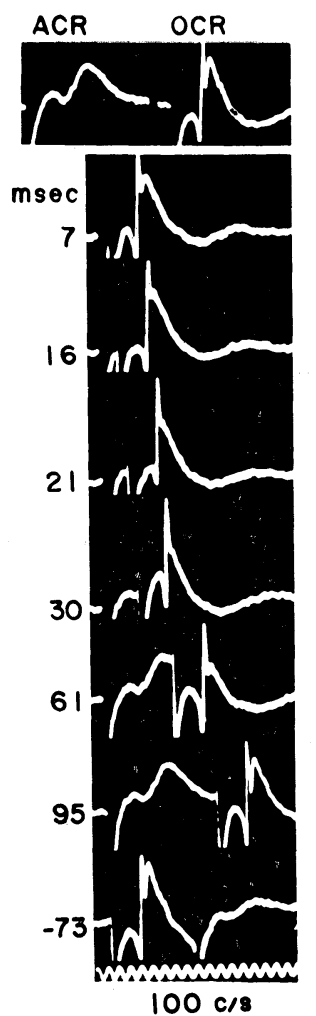

B

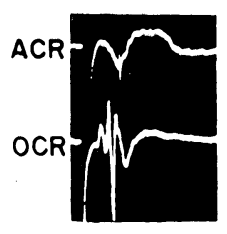

msec

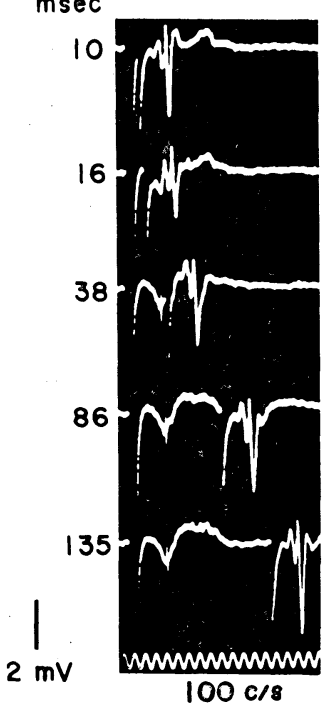

FIG. 5. Interaction between the antidromic cortical response (ACR) and the orthodromic cortical response (OCR). A : Top record shows the control of both responses led from the upper part of EPL (recording depth; $0.3 \mathrm{~mm}$ ). $\mathrm{B}$ : Top record shows the control of both responses in the lower part of EPL (recording depth; $0.4 \mathrm{~mm}$ ). Antidromic stimulation preceded orthodromic stimulation except in the lowest record of A where the stimulation sequence is reversed. Shock interval indicated beside each record in $\mathrm{msec}$. 
in FIG. 5. From the characteristics of the deep spike in the OCR as well as from the depth readings on the micromanipulator, $5 \mathrm{~A}$ was presumably led from the upper part and $5 \mathrm{~B}$ from the lower part of the external plexiform layer. Following an ACR, the amplitude of the deep spike of the OCR did not change in $5 \mathrm{~A}$, but clearly decreased in $5 \mathrm{~B}$. In both cases the following slow negativity in the OCR was abolished or attenuated during the slow negative excursion of the ACR. The lowest record in 5A, which was the only case in which orthodromic stimulation preceded antidromic stimulation, as indicated by the negative time value, simply showed as interaction in the reverse order; the slow negative excursion of the ACR was attenuated by a preceding OCR.

\section{DISCUSSION}

The orthodromic cortical potential recorded at the cortical surface consists of the initial and second slow potentials. These two potentials are dealt with in the works of ORREGO ${ }^{9,10)}$. The initial potential is due to the mass activity of the surface-running olfactory nerve axons. The slow potential, which ORREGO found to be maximal at the cell layer, is predominantly recorded in the upper layers, indicating the activity arising from glomerular dendrites. The deep spike occurs in the external plexiform layer, in which the apical dendritic shafts of the mitral cells have a vertical alignment. Slow recovery of the deep spike reaching up to $2 \mathrm{sec}$ or more is comparable to that of the slow potential in upper layer. This perhaps means that both potentials are produced postsynaptically in different portions of the same element, i.e. apical dendritic shaft and glomerular dendrite, then the excitability of the former is controlled by that of the latter. An electrode positioned perpendicular to the surface of the bulb will therefore pursue a course parallel to these elements as it is lowered into the bulb. It seems likely that the deep spike represents the activity of a few elements but not of a single one. With increasing recording depth the peak time and the latency of the deep spike do not change throughout most of the external plexiform layer. However, in this layer, a specific point is always seen at which the deep spike has a maximal amplitude suggesting the locus of generation. When approaching the mitral cell layer, the deep spike decreases usually in amplitude. From these findings we infer that the deep spike is generated in a limited area on the apical dendritic shaft of the mitral cell, and spreads electrotonically to a region close to the cell soma where unit spike discharges. A sharp positive phase at the deeper layer is a reflection of the deep negative spike. Next phase which sharply goes negative shows that the spikes, fired in somata or initial segments of mitral cells by the above mentioned electrotonic potential, conduct away along their axons.

Although not illustrated, the commissural cortical response (CCR) in the turtle is partially summatable with double shocks. $\mathrm{KERR}^{7)}$ has reported this 
response to be partially summatable in the rabbit although both WALSH ${ }^{14)}$ and $\mathrm{OCHI}^{8)}$, working in the same species, reported complete summation and identified the response as a postsynaptic potential induced in the granular cells via the anterior commissural fibers. Although the results reported here differ in some details from those of both $\mathrm{WALSH}^{14)}$ and $\mathrm{OCHI}^{8)}$, we think the $\mathrm{CCR}$ was evoked through the anterior commissure, as is definitely the case in mammals, rather than through multi-synaptic pathways described by ORREGO ${ }^{11)}$ via the pyriform cortices, for which the latency is too short. The granular cells send axons up into the region of the glomeruli, basal dendrites and somata of the mitral cells and, as reported by BAUMGARTEN et al. ${ }^{1}$, exert an inhibitory influence at these levels. Our own unpublished evidence confirms this observation.

Antidromic cortical response (ACR) can be broken down into separate. components. The first component, the grouped spikes, which is recorded in the internal plexiform layer, is apparently the result of asynchronous arrival of antidromic impulses in the mitral cell axons. The absence of interaction. between these spikes and the response to contralateral stimulation would indicate that this component does not involve dendritic activation in any way. The next component, the unit discharge, is recorded in the mitral cell layer and is presumably a mitral cell discharge since no other large cell types are seen at this level. The slow negativity of the ACR interacts with the CCR. This indicates that the slow negative excursion is the results of depolarization. of the mitral cell basal dendrites and of the activities of granular cells which synapse with the mitral cell dendrites.

Since activation from the opposite bulb fails to inhibit the deep spike, while it inhibits mitral cell soma response ${ }^{15)}$ this spike must arise along the dendritic shaft, where no granular cells or other endings make synaptic contact. From micromanipulator readings the region of maximal deep spike amplitude appears to be in the middle portion of the apical dendritic shaft. As suggested by the recent work of SPENCER and KANDEL ${ }^{13)}$ in the hippocampus, it is possible that the spike originates at the bifurcation point of the apical dendrite which is situated at approximately this depth. The deep spike is postulated to be a graded response; its slight decrease in the present case might be explained to some degree by inhibitory action of granular cells on the depolarization at the glomerulus.

Antidromic responses from pyriform cortex stimulation are attenuated significantly (FIG. 5B) when the electrode tip is deep, but not when the electrode is placed more shallowly (FIG. 5A). However, at all levels a preceding ACR will depress the slow negativity of the OCR and vice versa. The interaction between the slow negativities in both responses probably indicates that both occur in the basal dendrites of the mitral cell and the granular cells.

The first unit discharges observed, of short latency, must be produced in the mitral cell soma. It is maximal below the position where the deep spike 
is most prominent, and is presumably set off by the deep spike; but not at the same locus, as CRAGG and HAMLIN ${ }^{3)}$ reported for their material. The longer latency unit discharge would appear to originate from another mitral cell which is located deeper. A further study of more elaborate unit response patterns following stimulation of various paths will be presented in a following paper.

From the combined spatial and temporal sequences we infer the following sequence of responses in the secondary neurone of the bulb. The slow potential of the superficial dendritic terminals evokes the deep spike electrotonically along the dendritic shaft downwards; the spike spreads electrotonically to activate a spike in the mitral cell. A further slow process is set off in the basal dendrites and the granular cells via recurrent collaterals of the mitral cell axon. An antidromic response of the mitral cell does not directly reach the region of origin of the deep spike, though it may act on the basal dendrites and granular cell through recurrent collaterals.

\section{SUMMARY}

The orthodromic cortical response induced by the olfactory nerve stimulation was analysed by the depth recording and also the commissural and the antidromic stimulations. The following results were obtained;

1. The orthodromic cortical response (OCR) consists of two components: the initial potential and the slow potential, negative in sign, at the cortical surface. When recording in the external plexiform layer, the negative deep spike of $3-5 \mathrm{msec}$ duration occurred, following by the slow potential. The latency is about $15 \mathrm{msec}$ from the onset of the initial potential and remains constant throughout the external plexiform layer. The recovery process of deep spike is extremely slow, requiring $2 \mathrm{sec}$ or more for full recovery. In the deeper layer, unit discharge appeared.

2. In the internal granular layer the commissural cortical response (CCR) consists of positive-negative slow potential having a long latency of $60 \mathrm{msec}$. The deep spike does not interact with the preceding CCR.

3. The antidromic stimulation induces the grouped spike discharges and slow potential in the depth recording. The both negative slow potentials of OCR and antidromic cortical response $(A C R)$ are depressed by the commissural stimulation, but the deep spike of OCR and the grouped spikes of ACR do not interact with the commissural stimulation.

From the above findings, it seems likely that the deep spike is generated in the radially oriented secondary element, namely the apical dendrites of the mitral cell.

The authors wish to express their gratitude to Professors Rita Levi-Montalcini and G.H. Bishop for thier valuable criticism and encouragement throughout this work. We also wish to thank Dr. T. UcHIDA for the preparation of the manuscript. 
This research was supported by National Institute of Health Grant B-1602.

\section{REFERENCES}

1) Baumgarten, R. von, Green, J. D. And Mancia, M. Recurrent inhibition in the olfactory bulb. II. Effects of antidromic stimulation of commissural fibers. $J$. Neurophysiol., 25 : 489-500, 1962.

2) Baumgarten, R. von, Green, J. D. and Mancia, M. Slow waves in the olfactory bulb and their relation to unitary discharges. Electroenceph. clin. Neurophysiol., 14: 621-634, 1962.

3) CRAGG, B. G. AND HAmlyn, L.H. Action potentials of the pyramidal neurons of the hippocampus of the rabbit. J. Physiol., London, 129:608-627, 1955.

4) Crosby, E.C. And Humphrey, T. The nuclear configuration of the olfactory and accessory olfactory formulations and the nucleus olfactorius anterior of certain reptiles, birds and mammals. J. comp. Neurol., $71: 121-213,1939$.

5) Iwase, Y. ANd Uruha, M. Olfactory bulb response of rabbit. Science, 133 : 884885, 1961.

6) Iwase, Y., Uruha, M. And Ochi, J. Analysis of the olfactory bulb response induced by direct electrical stimulation in the rabbit Jap. J. Physiol., 11: 13-22, 1961 ,

7) Kerr, D. I. B. Properties of the olfactory system. Aust. J. exp. Biol., 38: 29-36, 1960.

8) Oсні, J. Olfactory bulb response to antidromic olfactory tract stimulation in the rabbit. Jap. J. Physiol., 13 : 113-128, 1963.

9) OrRego, F. The reptilian forebrain. I. The olfactory pathways and cortical area in the turtle. Arch. ital. Biol., 99: 425-445, 1961.

10) OrRego, F. The reptilian forebrain. II. Electrical activity in the olfactory bulb. Arch. ital. Biol., 99 : 446-465, 1961.

11) ORREgo, F. The reptilian forebrain. III. Cross connections between the olfactory bulbs and the cortical areas in the turtle. Arch. ital. Biol., 100:1-16, 1962.

12) Ottoson, D. Olfactory bulb potentials induced by electrical stimulation of the nasal mucosa in the frog. Acta Physiol. scand., 47: 160-172, 1959.

13) Spencer, W.A. And Kandel, E. R. Electrophysiology of hippocampal neurons. IV. Fast prepotentials. J. Neurophysiol., 24 : 272-285, 1961.

14) Walsh, R.R. Olfactory bulb potentials evoked by electrical stimulation of the contralateral bulb. Am. J. Physiol., 196: 327-329, 1959.

15) Yamamoto, C. Olfactory bulb potentials to electrical stimulation of the olfactory mucosa. Jap. J. Physiol., 11: 545-554, 1961. 